\title{
Natural killer frequency determines natural killer cytotoxicity directly in accentuated zones and indirectly in "moderate-to-normal frequency" segment
}

\author{
BORIS V. DONS' KOI', VICTOR P. CHERNYSHOV', DARIIA V. OSYPCHUK', IRINA SUDOMA ${ }^{2}$, \\ KSENIIA G. KHAZHYLENKO 3 , GALINA V. STRELKO ${ }^{4}$, WERA J. SIRENKO \\ ${ }^{1}$ Institute of Pediatrics, Obstetrics and Gynecology, National Academy of Medical Sciences of Ukraine, Kyiv, Ukraine \\ ${ }^{2}$ NADIYA Clinic, Kyiv, Ukraine \\ ${ }^{3}$ Isida-IVF, Kyiv, Ukraine \\ ${ }^{4}$ IVMED Fertility Centre, Kyiv, Ukraine \\ ${ }^{5}$ Institute for Reproductive Medicine-IVF, Kyiv, Ukraine
}

\begin{abstract}
Natural killer (NK) frequency and NK cytotoxicity (NKC) are key determining factors of a clinical outcome. In our previous study, we showed the prognostic clinical significance of immune parameters when they are beyond the optimal range (accentuated). In this study, we attempted to explain the disparity of accentuated but physiologically and immunologically normal NK parameters that might serve as negative clinical prognostics indications of failed pregnancies. We have analyzed NK\%, NKc levels, and their reciprocal correlation in 2,804 patients with reproductive failures.

In the entire clinical population, NK\% correlates with $N K c$. Interestingly, we found this relationship to be strongly dependent on NK level's status. NK\%-NKc correlation was the strongest $(r=0.2021$, $p<0.0001)$ in a patient group with high NK\% (> 17.5\%). Patients with NK\% between 15-17.5\% manifested lower but still significant correlation NK\%-NKc $(r=0.1213, p=0.0155)$. Additionally, significant correlation $(r=0.2689, p<<0.0001)$ between NK\% and NKc was observed in a group of patients with NK levels < 7\% (1.7-7\%). While patients' groups with NK\% (7-15\%) did not reveal NK\%-NKc association.

This led us to hypothesize that the qualitative-quantitative status of NK population is responsible for their cytotoxic activity. Consistent with our hypothesis, the "balanced zone" NK\% is tightly controlled, and thus does not correlate directly with NKc. In contrast, the "accentuated zones" of NK\% escape this control and directly affecting NKc. Demonstrated phenomena supports our idea about the clinical significance of immune accentuation and explains its novel physiological role.
\end{abstract}

Key words: NK cell cytotoxicity, NK cells, immune regulation.

(Cent Eur J Immunol 2020; 45 (3): 315-324)

\section{Introduction}

Natural killer cells (NK cells) are a subset of non-B and non-T peripheral blood lymphocytes, which appear to play a crucial role in the human innate immune responses. NK cells represent an "evolutionary bridge" between innate and adaptive immunity $[1,2]$. They mediate a cell contact-dependent cytolysis of target cells, including those expressing foreign major histocompatibility complex (MHC) molecules, immunoglobulin-bound antigens, virally infected cells, and tumor cells. The function of NK cells is important for clearance of tumor cells, removal of immunoglobulin-bound antigens, and for controlling viral infections [3, 4].

The role of NK cells in reproductive processes, such as implantation, trophoblast invasion, and spiral artery remodeling is critical, but still poorly understood [5]. The accumulating evidence links elevation of NK cells with unfavorable pregnancy outcomes in women [6-12]. NK function has been reported to be decreased in healthy pregnant women, persons with primary immunodeficiencies, and those with late-stage human immunodeficiency virus infections [13-15]. Resent progress in development of bet- 
ter methods for quantitative and functional assessment of NK cells might help to define their role in reproductive development and other clinically important mechanisms. For example, NK cell activity has already been established as an important indicator for predicting patient outcomes in certain cases [13-16].

Peripheral blood NK lymphocytes are a highly heterogeneous population of cells that differ by expression of surface ligands, a repertoire of KIR receptors, ability to secrete cytokines, and cytotoxic responses. All these NK cells share the ability to express CD56 in the absence of CD3 [17, 18]. Therefore, the heterogeneity of NK subsets is the major contributor to their multi-functionality. This population is named after the cytotoxic reaction measured by the NK assay against HLA-negative K562 cell line, yet only a part of NK cells manifests cytotoxicity. In our previous study, we demonstrated that only a subset of peripheral blood NK lymphocytes is able to respond to the K562, and that only a fraction of this subset determines levels of NK cytotoxicity in the entire population [19]. The amount of active NK cells varies from individual to individual, and their function can be easily modulated. However, this very group of cells tightly correlates with the actual NK cytotoxicity [20]. We also showed that balanced "optimal" counts of this fraction strongly correlate with successful embryo implantation in IVF stimulation cycle. Although, misbalance of NK activity is (decreased or increased active NK counts) negatively impacts the embryo implantation in IVF patients [16]. Interestingly, total NK cell counts did not appear to correlate with such clinical outcomes. Instead, NK quantity seemed to convert to NKc indirectly via a functional subset of active NK cells "the NK activity coefficient".

In the present study, we analyzed the effect of various levels of correlation between NK cytotoxicity and NK frequency, depending on NK cytotoxicity and NK lymphocytes levels-status.

\section{Material and methods}

\section{Study design}

In this retrospective study, we analyzed our laboratory data of 2,804 women, who underwent routine immunology investigation after IVF pregnancy failure. Data was acquired from March 2008 till October 2015 (1-3 months after last IVF cycle or 3-6 months after last pregnancy failure). The patients were under 39 years (average, 31.2) and had at least 1 episode of idiopathic IVF failure (average, 2.32) or at least 1 episode of idiopathic first trimester pregnancy failure (average, 2.04). The patients had no supplemental treatment with immunoglobulin or corticosteroids in the last 3 months. None of the patients had an active autoimmune disease or a history of autoimmune disease, and no patients had an active infection within at least
3 weeks. All of the study subjects signed an informed consent form before being enrolled into the study (approved by the Biomedical Ethics Committee of Institute of Pediatrics, Obstetrics, and Gynecology, National Academy of Medical Sciences of Ukraine, No. 6 dated December 17, 2012, and Clinical Immunology and Allergology Committee of Ministry of Health, No. 11 dated February 14, 2013, according to Declaration of Helsinki). Peripheral venous blood samples were drawn by venipuncture, and immunological studies (NK frequency and NK cytotoxicity) were performed in the Laboratory of Immunology, Institute of Pediatrics, Obstetrics, and Gynecology of the National Academy of Medical Sciences, Kyiv, Ukraine.

For NK cytotoxicity distribution, we analyzed our laboratory data of 9,535 individuals from similar clinical group. In isolated study for comparison of NKc data, we analyzed 823 individuals' data, where NKc was measured by TDA relies assay. All investigations were made in $5 \mathrm{~h}$ period after obtaining blood samples.

\section{Flow cytometry of lymphocyte subsets}

Lymphocyte subsets were identified by three-color flow cytometry, using erythrocyte-lysing whole blood method of lymphocyte staining by FITC-, PE-, or PE-Cy5-conjugated monoclonal antibodies (BD Bioscience, San Jose, USA). Stained samples were lysed, washed, and analyzed by FACScan flow cytometer, using CellQuest software (BD Bioscience, San Jose, USA). NK lymphocyte was calculated as the percentage of CD3CD56+ lymphocyte (include CD56++ subsets).

\section{NK cytotoxicity assays}

Briefly, a total of $2 \times 10^{6}$ PBL purified from peripheral blood of patients by Histopaque-1077 (Sigma-Aldrich, MO, USA) gradient were washed in PBS. Flow cytometry NK cytotoxicity assay (FCA) was described previously [20]. We used CMFDA (Molecular Probes, Eugene, OR, USA) for labeling K562. After $4 \mathrm{~h}$ of incubation, dead cells were labeled by PI. Less than 5\% spontaneous lysis of target cells was observed in these experiments. We used 50\% permeabelized K562 culture for targets-gate correction. $100 \mu \mathrm{l}$ target cell was killed by $50 \mu \mathrm{l}$ ethanol (96\%). After $10 \mathrm{sec}$ wortexing, $100 \mu \mathrm{l}$ of PBS and $100 \mu \mathrm{l}$ of unaffected targets were added. The suspension obtained consisted of $50 \%$ of alive and $50 \%$ of permeable cells.

\section{TDA release NK cytotoxicity assay}

TDA release NK cytotoxicity assay (TDARA) was described previously [20]. Targets K562 (a human erythroleukemia cell line) in RPMI 1640, supplemented with $10 \%$ bovine serum were labeled at concentration of $1 \times 10^{6}$ cells/ml, with $5 \mu$ BATDA reagent (bis acetooxymethyl 2,2' : 6',2',-terpyridine-6,6' '-dicarboxylate, PerkinElmer Wallac, Turku, Finland) for $20 \mathrm{~min}$ at $37^{\circ} \mathrm{C}$. Labeled cells 
were washed three times in RPMI/10\% bovine serum and incubated with PBMC at various effector/target ratio (E : T $20: 1,10: 1,5: 1)$. After co-incubation of the effector and target cells for $4 \mathrm{~h}$ at $37^{\circ} \mathrm{C}$ in a humidified $5 \% \mathrm{CO}_{2}$ incubator, the cells were mixed and centrifuged, and $20 \mu \mathrm{l}$ of the supernatant was mixed with $180 \mu \mathrm{l}$ europium solution (PerkinElmer Wallac, Turku, Finland) and incubated for $15 \mathrm{~min}$ at room temperature, while shaking in a flat-bottom 96-well plate (Nunc, Denmark). Time-resolved fluorometry was carried out with ARCUS (Wallac Oy, Turku, Finland). The percentage of specific lysis was calculated as ((experimental release-spontaneous release) / $($ maximal release-spontaneous release $) \times 100 \%$. The maximal release was determined by addition of Triton X100 in a final concentration of $0.25 \%$. The assay was performed in quadruplicate for each $\mathrm{E}: \mathrm{T}$ ratios.

For both assay individual, cytotoxic activity was calculated as $\%$ of cytotoxicity (E: T ratio $15: 1$ as average from levels at $20: 1$ and $10: 1)$.

Intra-laboratory normal levels were obtained from $90 \%$ CI calculated for 74 healthy fertile women with subsequent normal pregnancy development [11].

\section{Statistical analysis}

The statistical analysis of the results was performed using Fisher's exact test (unpaired, non-parametric, two-sided p-value) and Spearman's and Pearson's correlation (InStat version 3.0 for Windows GraphPad Software Inc., San Diego, CA, USA).

\section{Results}

\section{Distribution of values}

We analyzed the distribution of NK lymphocyte levels in 2,804 patients and determined the average counts to be $12 \%$, typical for healthy Caucasian women [21, 22]. Only $1.9 \%(55 / 2,804)$ of patients had NK levels $>26.1 \%$, and $4.6 \%(131 / 2,804)$ patients had NK levels $<4.8 \%$ (Fig. 1A). According to our clinical normal reference $[1,23]$ for patients with favorable implantation and pregnancy prognosis $(5.5-21 \%)$, the decreased NK\% was observed in $5.1 \%(144 / 2,804)$ patients and increased NK\% in $6.2 \%(174 / 2,804)$ of the investigated patients' population.

Distribution of NKc and NKc average levels was comparable to published results from other groups [13, 24], with a notable trend towards increased and decreased values. According to our clinical references for patients with favorable implantation and pregnancy prognosis [11, 23], we showed that $13.1 \%(369 / 2,804)$ of patients presented decreased NKc, whereas $19.4 \%$ of patients $(545 / 2,804)$ had increased NKc levels (Fig. 1B).

NKc was elevated in $20.7 \%$ (51/246) of patients with 1 episode of reproductive failure (pregnancy or IVF failure) in contrast to $34.5 \%$ and $31.3 \%$ of patients with 2 and more
IVF failure (297/843), or 2 and more pregnancy failure $(316 / 1,008)$ episodes.

\section{Correlations}

Consistently with our previous reports [20], the frequency of NK lymphocytes in the whole clinical population strongly correlated with NK cytotoxicity $(r=0.4268$, $p<0.0001$ ) (Fig. 2 and Table 1). Also, we did not find a significant correlation between NK counts and NK cytotoxicity in the group of patients with "normal NK levels". In contrast, this correlation was strong in patients' groups with decreased or increased NK frequency. To investigate this phenomenon closer, we divided the population into groups $(n=400)$ according to the individual NK lymphocyte counts. We found strong NK\%-NKc correlation $(r=0.2021, p<0.0001)$ in a group of patients with high NK\% (> 17.5\%) (Table 1). Lower but also significant correlation was observed in patients with $\mathrm{NK} \%$ between $15 \%$ and $17.5 \%(r=0.1213, p=0.0155)$. The absence of NK\%NKc correlation was observed in the patient groups with following NK\% (12-15\%), (12-10\%), (10-8\%), and (8-7\%). However, strong correlation $(r=0.2689, p<0.0001)$ between $\mathrm{NK} \%$-NKc re-appeared in the patients' group with NK levels $<7 \%$ (1.7-7\%) (Table 1).

These findings agree with data obtained using an alternative NKc assay (Eu-Badta). In contrast to FC NK assay, where dead cell targets became permeable for PI, this modification measured cytotoxicity as Badta reagent release from lysing targets. Similarly, to the study using FC NK assay, we grouped patients according to NK lymphocyte levels (each group of approximately 200 patients). Our data analysis revealed significant NK\%-NKc correlation in patients' groups with NK\% $<7.5 \%(r=0.212, p=0.0026)$ and $>15 \%(r=0.2596, p<0.0001)$. In patients with NK\% $(7.5-10.5 \%)$ and $(10.5-15 \%)$, NK\%-NKc correlation was not significant $(r=0.123, p=0.08)$ and $(r=0.14$, $p=0.07)$, respectively. Therefore, we showed that NK\%NKc correlation depends on NK frequency status.

Next, we wanted to determine whether NKc-NK\% correlation may depend on NK cytotoxicity status. Similar to our previous analysis, we investigated NK\%-NKc correlation in patients grouped according to their NKc score. Significant correlations were observed in groups with accentuated/elevated $(>45.5 \%)$ or downregulated $(<13 \%)$ NKc at E : T ratio of $15: 1(r=0.2725, p<0.0001)$ and $(r=0.1707, p=0.0006)$, respectively. In groups with moderate NKc levels (> 13 and $<45.5$ ), no significant correlation was found (Table 2).

Here, we demonstrated that variation of quality status of NK population plays an important role in correlative association with their cytotoxic activity. Therefore, the quantitative changes within accentuated zones affect the quality of associative character in $\mathrm{NK} \%-\mathrm{NKc}$ and its impact on clinical outcomes. 

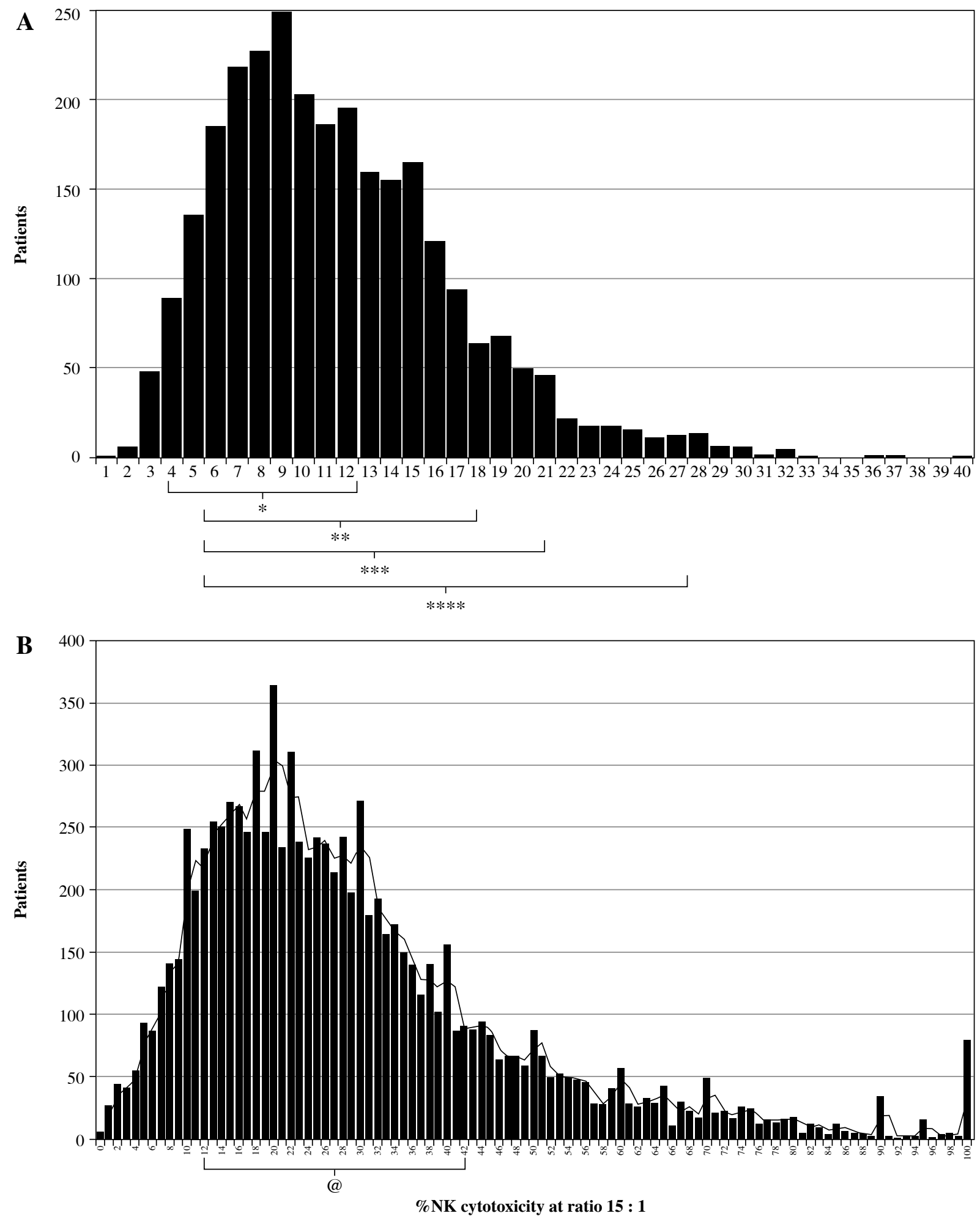

Fig. 1. (A) Distribution of levels natural killer (NK) lymphocytes and (B) NK cytotoxicity in patients' groups. A) NK (CD56+CD3-) cells \% from all lymphocytes $(n=2,804)$, B) NK cytotoxicity (E : T, $15: 1)$ was measured by FC NK assay against $\mathrm{K} 562(n=9,535)$. * normal value for pregnancy prognosis $(3-12 \%)$ [6], ** normal value for pregnancy prognosis $(5-18 \%)$ [7], *** our laboratory favorable value for implantation and pregnancy prognosis $(5.5-21 \%)$ [11], **** reference value for young Caucasian women (4.8-26.1\%) [38], @ our laboratory favorable value for implantation and pregnancy prognosis $(12.5-41 \%)$ [11] 


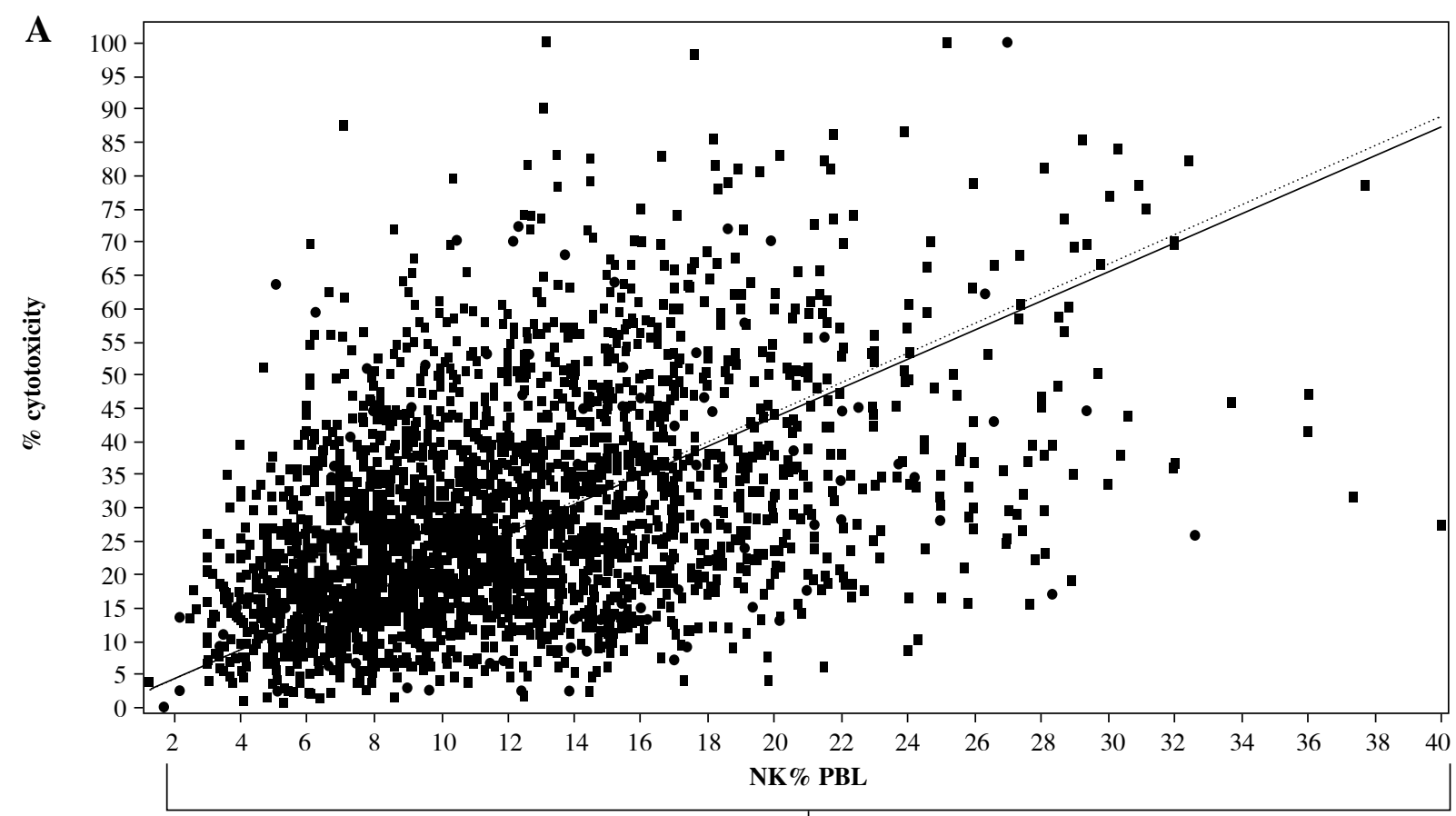

$r=0.4268, p$-value $<0.0001$ is considered extremely significant

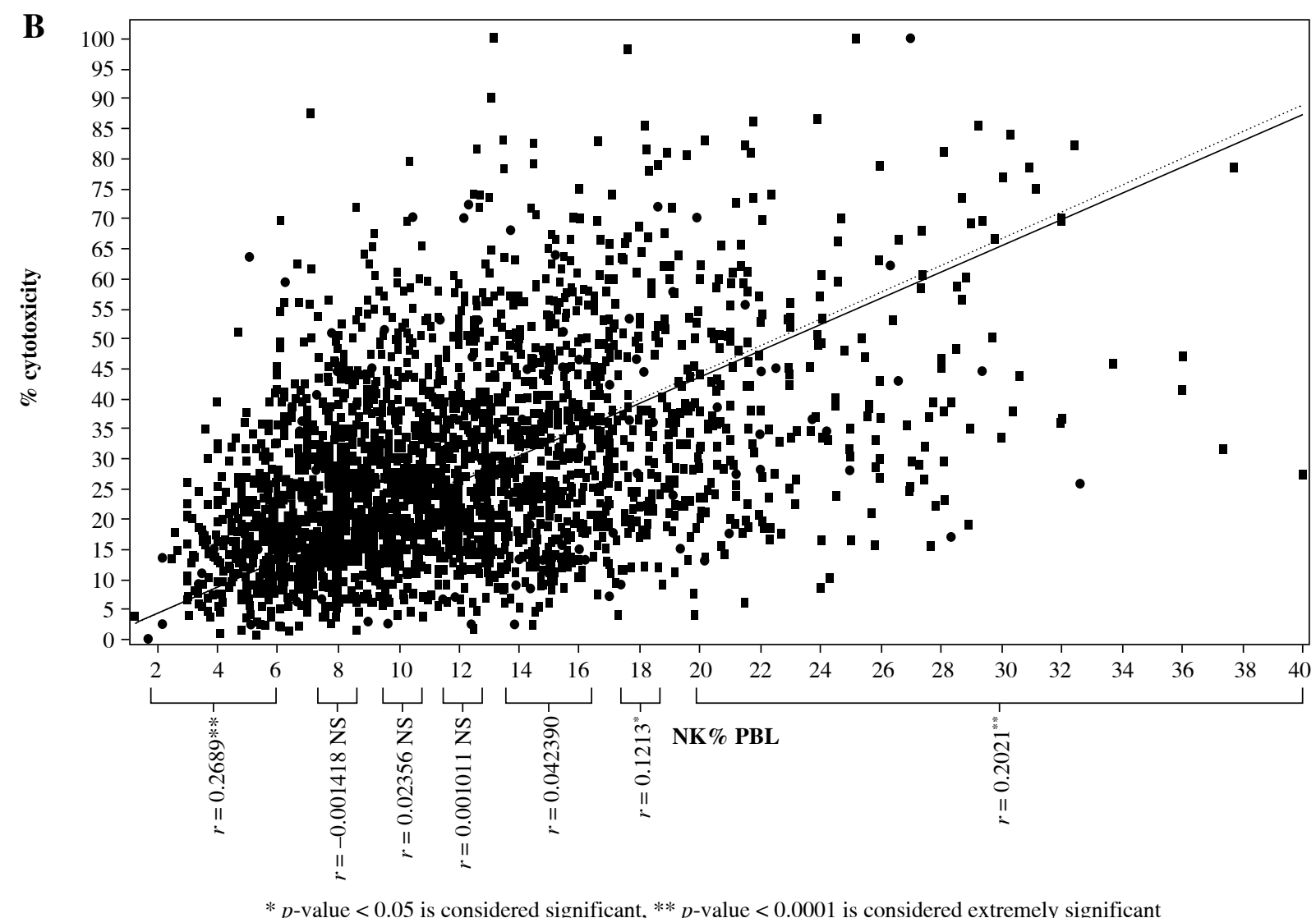

Fig. 2. A) Correlation of natural killer (NK) frequency with NK cytotoxicity in whole population $(n=2,804)$ and $(\mathbf{B})$ in separate zones of NK\% distributions 
Table 1. Correlation between natural killer (NK) frequency and NK cytotoxicity in groups divided according to NK numbers

\begin{tabular}{|c|c|c|c|c|c|}
\hline $\begin{array}{l}\text { \% NK lymphocyte } \\
\text { (CD3negCD56pos) }\end{array}$ & $n$ & $\begin{array}{l}\text { Correlation } \\
\text { coefficient }(\mathbf{r})\end{array}$ & $r^{2}$ & $\begin{array}{l}\text { SD of residuals } \\
\text { from line }(S y \cdot x)\end{array}$ & $p$-value \\
\hline All (1.7-40) & 2,804 & 0.4268 & 0.1822 & 15.844 & $<0.0001^{*}$ \\
\hline$<7(1.7-7)$ & 453 & 0.2689 & 0.07231 & 1.029 & $<0.0001 *$ \\
\hline $7-8$ & 257 & -0.001418 & $2.010 \times 10^{6}$ & 0.4992 & 0.9775 \\
\hline $8-10$ & 461 & 0.02356 & 0.0005553 & 0.5009 & 0.6384 \\
\hline $10-12$ & 425 & 0.001011 & $1.022 \times 10^{6}$ & 0.6714 & 0.9839 \\
\hline $10-15$ & 461 & 0.04239 & 0.001797 & 0.7441 & 0.3984 \\
\hline $15-17.5$ & 347 & 0.1213 & 0.01470 & 0.8482 & $0.0155^{* * *}$ \\
\hline$>17.5(17.5-40)$ & 400 & 0.2021 & 0.04083 & 19.613 & $<0.0001 *$ \\
\hline
\end{tabular}

Table 2. Correlation between natural killer (NK) cytotoxicity and NK frequency in group divided according to NK cytotoxicity levels

\begin{tabular}{lccccc}
\hline $\begin{array}{l}\text { \%NK cytotoxicity at E : T } \\
\text { ratio 15 : }\end{array}$ & $\boldsymbol{n}$ & $\begin{array}{c}\text { Correlation } \\
\text { coefficient }(\boldsymbol{r})\end{array}$ & $\boldsymbol{r}^{2}$ & $\begin{array}{c}\text { SD of residuals } \\
\text { from line (Sy.x) }\end{array}$ & $\boldsymbol{p}_{\text {-value }}$ \\
\hline All $(0-100)$ & 2,804 & 0.4268 & 0.1822 & 15.844 & $<0.0001^{*}$ \\
\hline$<13(0-13)$ & 396 & 0.1707 & 0.02913 & 3.009 & $0.0006^{*}$ \\
\hline $13-18$ & 403 & 0.08541 & 0.007295 & 1.427 & 0.0892 \\
\hline $18-23$ & 401 & 0.04866 & 0.002368 & 1.405 & 0.3233 \\
\hline $23-28.5$ & 404 & 0.01114 & 0.0001241 & 1.541 & 0.8212 \\
\hline $28.5-35$ & 404 & 0.09128 & 0.008332 & 1.933 & 0.0661 \\
\hline $35-45.5$ & 398 & 0.02191 & 0.0004802 & 3.024 & 0.6590 \\
\hline$>45.5$ (45.5-100) & 398 & 0.2725 & 0.07424 & 10.319 & $<0.0001^{*}$ \\
\hline$*$ considered extremely significant & & & & &
\end{tabular}

\section{Discussion}

NK frequency and NK cytotoxicity are significant parameters that determine clinical outcomes. Recently, several independent groups showed that individuals with elevated NK lymphocyte levels and NK cytotoxicity present lower risk and better clinical outcomes in viral infections and oncology diseases, compared to patients with lower NK\% and NKc levels [25-29]. In contrast, increased NK levels after ischemic stroke have been associated with increased risk of sepsis [30]. Also, elevated NK activation correlates with liver injury in chronic hepatitis B patients [31]. In transplantation patients, NK role was shown to be ambiguous, according to recent reports [32, 33]. In these studies, NK cells exacerbated $\mathrm{T}$ cell responses during transplant rejection, but also promoted tolerance induction under immunosuppression. On animal model, NK cells play a critical role in cardiac allograft vasculopathy and long-term kidney allograft injury [34, 35]. Growing amount of evidence [6-12] suggests a link between increased $\mathrm{NK} \%$ and $\mathrm{NKc}$ and reproductive failure.
Despite these facts, the question still remains: what are the normal (optimum) levels of NK? NK\% varies depending on gender, ethnical, and racial backgrounds [36-39]. Moreover, NK\% and NKc levels are higher in males compared to females. This difference is significant in reproductive age. In females 40 and older, these differences become less pronounced [40, 41], while NK\% and NKc levels are markedly reduced in pregnant women. Not surprisingly, NK system attracts special interest in the field of immunology of reproduction. It is accepted that NK cells play a critical, yet poorly defined role in reproductive processes, such as implantation, trophoblast invasion, and spiral artery remodeling [5]. On the one hand, elevated NK cell frequency [7, 8] cytotoxicity [9] and imbalance between inhibitory and activating receptor expression on NK cells $[10,11]$ are associated with different reproductive failures [12]. On the other hand, NK cell activity is also necessary for reproduction; it was shown that fetal growth restriction is associated with reduced proportions of NK in the decidua basalis [42], and that NK cell depletion in mice leads to placental abnormalities [43], thickening of the spiral artery 
[44], and fetal loss [45]. At different times, several groups proposed to define "reproductive normal values" of NK frequency [7, 46, 47]. Following values were suggested as reference points $<12 \%,<15 \%$, or $<18 \%$, respectively, as favorable values for implantation and normal pregnancy outcome. In contrast, the elevated NK counts were suggested as indicators of increased risk of reproductive failure in women.

Undoubtedly, the predictability of prognosis using NK frequency as well as the role of elevated NK\% in reproductive outcome are still up for debate [48, 49]. Many investigators agree that measurement of the functional activity of NK cells is more important for clinical prognosis [1]. However, limited affordability, complexity, and lack of standardization were the main reasons that this marker is still not a part of the routine clinical diagnostics. In contrast, the characterization of NK phenotype markers is much easier and possesses comparable clinical utility. It was shown, for example, that misbalance between KIR receptor activation and ligand subsets is associated with reproduction failures $[48,50]$.

In this study, we demonstrated that different qualitative states of NK population have different correlative associations with cytotoxicity. The absence of correlation between NK\% and NK cytotoxicity was found in the moderate-to-normal zone of NK frequency. This could mean that in this zone, the counts of NK cells do not directly determine cytotoxicity. Instead, the NK lymphocytes counts in the moderate-to-normal zone are sufficient and are compatible with the generation of any NKc levels. Associations between $\mathrm{NK} \%$ and $\mathrm{NKc}$ are more quantitative in nature. Within the balanced "normal" zone, NK cytotoxicity levels rather than NK frequency is determined by proportions of active subsets. This regulatory mechanism adjusts NKc to the actual (necessary) situation, thus, reversing the role of NK frequency in these zones. Here, in the "normal zone", NK numbers are not directly equated to NKc. In contrast, accentuation of NK through inflammation-affectation results in decreased/increased NK numbers and deregulation of the clonal population balance. Affectation of regulatory mechanisms results in the direct impact of NK frequency on cytotoxicity. Therefore, the quality directly determines functional quantity. Hence, it is possible that increase or decrease in NK frequency is regulated largely through cytotoxic NK subsets proportions.

Increased NK\% and NKc correlation is a first unfavorable accentuation marker displayed during reproductive development [7]. Previously, we showed that some accentuations have negative predictive value for implantation and pregnancy outcome. The majority of those outcomes were associated with NK surface markers expression and their activity. Among them, NK accentuations possess bilateral features. Decreased as well as increased CD8, CD158 expression, and CD69 activation proved to be predictive of the negative clinical outcome [16, 23, 51]. In the case of accentuated T lymphocytes, negative predictive markers were increased (HLA-DR CD56, CD158) or decreased (CD4 levels) [52].

Analyzing the predictive significance of CD8 expression on NK, we demonstrated that elevated levels are associated with implantation failures, while decreased CD8 levels were associated with subsequent pregnancy failures. We also have shown that both accentuated phenotypes' individuals have a higher frequency of other accentuations compared to patients with balanced NK-CD8 expression [51].

In this investigation, we demonstrated that in addition to clinical manifestation, NK\% accentuations lead to a deregulation of NKc. Thus, NK frequency indirectly affects NK cytotoxicity through the regulation mechanisms and subsets balance. However, in the accentuated zones, this mechanism is not enough to correct and modulate physiological (necessary) conditions. A demonstrated phenomenon supports our idea about immune accentuation and sheds light on its physiological role.

In this study, we analyzed the levels of NK cells in patients with difficulties in reproduction. According to the levels of NK cells, the patients almost conformed to the known reference levels for young healthy women of the same ethnic group [21, 22]. Many studies reported higher levels of NK cells in healthy fertile women compared to IVF (in vitro fertilization) and RSA (recurrent spontaneous abortion) patients $[6-11,54]$. The probable explanation might lie in dissimilarity of reproductive state within the "somatic healthy" women group. In today's world and in our local population, most of women delay childbirth to a later point in their life. In recent years, the prevalence of sub-fertile and infertile states markedly increased. Most of these states are not associated with general health indicators and are hard to predict. Therefore, the likelihood of occurrence of such adverse states in some of the "somatic healthy" women at a future time point is relatively high. Additionally, IVF failure and RSA diagnoses are being made usually after three consecutive first-trimester pregnancy failures with the normal fetus (chromosomally and without pathology), or three consecutive IVF failures with a high quality of embryos. In our group, most patients chose not to wait for the confirmation of their clinical state and requested immunodiagnostics early in pregnancy. Thus, significant part of the study population had no definite diagnosis of idiotypic IVF failure and RSA. Similarly, the population labeled as the "somatically healthy" women were not formally confirmed as reproductively healthy. In fact, reproductive disturbances of the immune genesis are not often accredited to the general clinical status, but rather a situational combination of adverse immunological, physiological, and genetic factors. Therefore, it is not always true that the genetic mother-fetus combination and functional state of a female patient is a lasting independent predictive marker of unfavorable outcome. Moreover, the fertility can only be confirmed by the onset of pregnancy 
and carrying of pregnancy in the given cycle instead of the normal range of different parts of the immune and endocrine systems. Interestingly, the fact that the NK percentage is gaining a new role when passing a certain threshold, might help to determine the physiologically-balanced standard range for specific clinical groups. Our study demonstrates that by knowing this range, we might be able to predict and/or create more favorable corridor for the course of pregnancy.

NK cells testing is still a controversial issue in RM and RIF patients. This controversies start to be a separate topic of debates [53, 54].

In multivariate analysis [55], it was shown that blood NK cells expansion was an independent risk factor for RF (both recurrent miscarriages and implantation failures). Significance of this risk factor is growing with patient's age. In contrast, in same year and in similar patients' numbers was shown [56] that elevated pNK cell activity is not independent risk factor for subsequent miscarriage. In same investigation, untypical age-associations with subsequent miscarriage were observed. Older women (32-35 years old) have better pregnancy outcome than younger (30-31 years old). Also, it was reported that Japanese local population was practically devoid of HLA C2 carriers and predominantly presented KIR AA+ [57]. Frequency of risk pregnancy combination (fetal HLA C2+ with mother KIR AA) in this population was minimal, which could possibly reduce the significance of NK activity for clinical outcome.

\section{Conclusions}

It is possible that accentuated NK function form unfavorable background for reproduction, but this risk is realized indirectly through associations with other immune-endocrine factors, genetic deviations, or mother/ fetus combinations. It was observed [58] that NK cytotoxicity is the effector mechanism of placental dysfunctions in alloimmune thrombocytopenia model. Anti- $\beta 3$ integrin $\mathrm{ab}$ and endothelium cells activation was the origin of pathology that causes damage in placenta through NK cells cytotoxicity. Inhibitions of NK cytotoxic receptors completely revoke placenta dysfunction.

It is too early for a reliable NK cells testing in routine diagnostic, but at same time, it is too late to ignore these parameters in the formation of reproductive unfavorable conditions.

\section{The authors declare no conflict of interest.}

\section{References}

1. Whiteside TL, Herberman RB (1994): Role of human natural killer cells in health and disease. Clin Diagn Lab Immunol 1: 125-133.
2. Sun JC, Lanier LL (2009): Natural killer cells remember: An evolutionary bridge between innate and adaptive immunity. Eur J Immunol 39: 2059-2064.

3. Knorr D, Bachanova V, Michael RV, Miller JS (2014): Clinical utility of natural killer cells in cancer therapy and Transplantation. Semin Immunol 26: 161-172.

4. Kutukculer N, Azarsiz E, Karaca NE, et al. (2015): Clinical and Laboratory Approach to the Evaluation of Innate Immunity in Pediatric CVID Patients. Front Immunol 6: 145.

5. Le Bouteiller P, Costa EH, Aguerre-Girr M, Tabiasco J (2009): Immunity of pregnancy: novel concepts. Bull Acad Natl Med 193: 1029-1041.

6. Coulam CB, Goodman C, Roussev RG, et al. (1995): Systemic CD56+ cells can predict pregnancy outcome. Am J Reprod Immunol 33: 40-46.

7. Beer AE, Kwak JY, Ruiz JE (1996): Immunophenotypic profiles of peripheral blood lymphocytes in women with recurrent pregnancy losses and in infertile women with multiple failed in vitro fertilization cycles. Am J Reprod Immunol 35: 376-382.

8. Winger EE, Reed JL, Ashoush S, et al. (2011): Elevated Preconception CD56(+) 16(+) and/or Th1:Th2 Levels Predict Benefit from IVIG Therapy in Subfertile Women Undergoing IVF. Am J Reprod Immunol 66: 394-403.

9. Matsubayashi H, Hosaka T, Sugiyama Y, et al. (2001): Increased natural killer-cell activity is associated with infertile women. Am J Reprod Immunol 46: 318-322.

10. Ntrivals EI, Bowser CR, Kwak-Kim J, et al. (2005): Expression of killer immunoglobulin-like receptors on peripheral blood NK cell subsets of women with recurrent spontaneous abortions or implantation failures. Am J Reprod Immunol 53: 215-221.

11. Chernyshov VP, Sudoma IO, Dons'koi BV, et al. (2010): Elevated NK cell cytotoxicity, CD158a expression in NK cells and activated $\mathrm{T}$ lymphocytes in peripheral blood of women with IVF failures. Am J Reprod Immunol 64: 58-67.

12. Yamada H, Kato EH, Kobashi G, et al. (2001): High NK cell activity in Early Pregnancy correlates with subsequent abortion with normal chromosomes in women with recurrent abortion. Am J Reprod Immunol 46: 132-136.

13. Kane KL, Ashton FA, Schmitz JL, Folds JD (1996): Determination of natural killer cell function by flow cytometry. Clin Diagn Lab Immunol 3: 295-300.

14. Sun C, Sun HY, Xiao WH, et al. (2015): Natural killer cell dysfunction in hepatocellular carcinoma and NK cell-based immunotherapy. Acta Pharmacol Sin 36: 1191-1199

15. Golden-Mason L, Cox AL, Randall JA, et al. (2010): Increased natural killer cell cytotoxicity and NKp30 expression protects against hepatitis $\mathrm{C}$ virus infection in high-risk individuals and inhibits replication in vitro. Hepatology 52: 15811589.

16. Dons'koi BV, Chernyshov VP, Sirenko VY, et al. (2014): Peripheral blood natural killer cells activation status determined by CD69 upregulation predicts implantation outcome in IVF. Immunobiology 219: 167-171.

17. Di Santo JP (2006): Natural killer cell developmental pathways: a question of balance. Annu Rev Immunol 24: 257-286.

18. Lanier LL (2003): Natural killer cell receptor signaling. Curr Opin Immunol 15: 308-314.

19. Donskoǐ BV, Chernyshov VP, Osypchuk DV (2011): The immunophenotypic characteristics of two functionally different natural killer cell subpopulations in peripheral human blood. Fiziol Zh 57: 29-35. 
20. Dons'koi BV, Chernyshov VP, Osypchuk DV (2011): Measurement of NK activity in whole blood by the CD69 upregulation after co-incubation with K562, comparison with NK cytotoxicity assays and CD107a degranulation assay. J Immunol Methods 30: 187-195.

21. Reichert T, DeBruyčre M, Deneys V, et al. (1991): Lymphocyte subset reference ranges in adult Caucasians. Clin Immunol Immunopathol 60: 190-208.

22. Jentsch-Ullrich K, Koenigsmann M, Mohren M, Franke A (2005): Lymphocyte subsets' reference ranges in an ageand gender-balanced population of 100 healthy adults a monocentric German study. Clin Immunol 116: 192-197.

23. Chernyshov VP, Dons'koi BV, Sudoma IO, Goncharova YO (2014): Favorable immune phenotype predicts successful implantation and pregnancy. Immunol Lett 162: 217-221. Chung HJ, Park CJ, Lim JH, et al. (2010): Establishment of a reference interval for natural killer cell activity through flow cytometry and its clinical application in the diagnosis of hemophagocytic lymphohistiocytosis. Int J Lab Hematol 32: 239-247.

24. Sun C, Sun HY, Xiao WH, et al. (2015): Natural killer cell dysfunction in hepatocellular carcinoma and NK cell-based immunotherapy. Acta Pharmacol Sin 36: 1191-1199.

25. Imai K, Matsuyama S, Miyake S, et al. (2000): Natural cytotoxic activity of peripheral-blood lymphocytes and cancer incidence: an 11-year follow-up study of a general population. Lancet 356: 1795-1999.

26. Ogata K, An E, Shioi Y, et al. (2001): Association between natural killer cell activity and infection in immunologically normal elderly people. Clin Exp Immunol 124: 392-397.

27. Sansoni P, Cossarizza A, Brianti V (1993): Lymphocyte subsets and natural killer cell activity in healthy old people and centenarians. Blood 82: 2767-2773.

28. Ogata K, Yokose N, Tamura H (1997): Natural killer cells in the latedecades of human life. Clin Immunol Immunopathol 84: 269-275.

29. De Raedt S, De Vos A, Van Binst AM, et al. (2015): High natural killer cell number might identify stroke patients at risk of developing infections. Neurol Neuroimmunol Neuroinflamm 2: e71.

30. Zheng Q, Zhu YY, Chen J, et al. (2015): Activated natural killer cells accelerate liver damage in patients with chronic hepatitis B virus infection. Clin Exp Immunol 180: 499-508.

31. Villard J (2011): The role of natural killer cells in human solid organ and tissue transplantation. J Innate Immun 3: 395-402.

32. Crespo M, Yelamos J, Redondo D, et al. (2015): Circulating NK-cell subsets in renal allograft recipients with anti-HLA donor-specific antibodies. Am J Transplant 15: 806-814.

33. Zhang ZX, Huang X, Jiang J, et al. (2015): Natural Killer Cells Mediate Long-term Kidney Allograft Injury. Transplantation 99: 916-924.

34. Zhang ZX, Huang X, Jiang J, et al. (2014): Transplantation. Natural killer cells play a critical role in cardiac allograft vasculopathy in an interleukin-6-dependent manner. Transplantation 98: 1029-1039.

35. Choi J, Lee SJ, Lee YA, et al. (2014): Reference values for peripheral blood lymphocyte subsets in a healthy korean population. Immune Netw 14: 289-295.

36. Torres AJ, Cisneiros P, Guedes R, et al. (2015): Lymphocyte subset reference intervals in blood donors from northeastern Brazil. An Acad Bras Cienc 87: 1019-1025.

37. Bisset LR, Lung TL, Kaelin M, et al. (2004): Reference values for peripheral blood lymphocyte phenotypes applicable to the healthy adult population in Switzerland. Eur J Haematol 72: 203-212.

38. Reichert T, DeBruyčre M, Deneys V, et al. (1991): Lymphocyte subset reference ranges in adult Caucasians. Clin Immunol Immunopathol 60: 190-208.

39. Jentsch-Ullrich K, Koenigsmann M, Mohren M, Franke A (2005): Lymphocyte subsets' reference ranges in an ageand gender-balanced population of 100 healthy adults a monocentric German study. Clin Immunol 116: 192-197.

40. Chng WJ, Tan GB, Kuperan P (2004): Establishment of Adult Peripheral Blood Lymphocyte Subset Reference Range for an Asian Population by Single-Platform Flow Cytometry: Influence of Age, Sex, and Race and Comparison with Other Published Studies. 2004. Clin Diagn Lab Immunol 11: 168-173.

41. Eide IP, Rolfseng T, Isaksen CV, et al. (2006): Serious foetal growth restriction is associated with reduced proportions of natural killer cells in decidua basalis. Virchows Arch 448: 269-276.

42. Barber EM, Pollard JW (2003): The uterine NK cell population requires IL-15 but these cells are not required for pregnancy nor the resolution of a Listeria monocytogenes infection. J Immunol 171: 37-46.

43. Croy BA, Zhang J, Tayade C, et al. (2010): Analysis of uterine natural killer cells in mice. Methods Mol Biol 612: 465-503.

44. Guimond MJ, Luross JA, Wang B, et al. (1997): Absence of natural killer cells during murine pregnancy is associated with reproductive compromise in TgE26 mice. Biol Reprod 56: 169-179.

45. Coulam CB, Goodman C (2000): Increased pregnancy rates after IVF/ET with intravenous immunoglobulin treatment in women with elevated circulating C56+ cells. Early Pregnancy 4: $90-98$.

46. Karami N, Boroujerdnia MG, Nikbakht R, Khodadadi A (2012): Enhancement of peripheral blood CD56(dim) cell and NK cell cytotoxicity in women with recurrent spontaneous abortion or in vitro fertilization failure. J Reprod Immunol 95: 87-92.

47. Thum MY, Bhaskaran S, Abdalla HI, et al. (2004): An increase in the absolute count of CD56dimCD16+CD69+ NK cells in the peripheral blood is associated with a poorer IVF treatment and pregnancy outcome. Hum Reprod; 19: 23952400.

48. Thum MY, Bhaskaran S, Bansal AS, et al. (2005): Simple enumerations of peripheral blood natural killer (CD56+ NK) cells, B cells and T cells have no predictive value in IVF treatment outcome. Hum Reprod 20: 1272-1276.

49. Lee SK, Na BJ, Kim JY, et al. (2013): Determination of clinical cellular immune markers in women with recurrent pregnancy loss. Am J Reprod Immunol 70: 398-411.

50. Dons'koi BV (2015): Accentuated hypo- and hyper-NK lymphocyte CD8 expression is a marker of NK subsets' misbalance and is predictive for reproductive failures. Immunobiology 220: 649-655

51. Dons'koǐ BV, Chernyshov VP, Sudoma IO, et al. (2013): Qualitative analysis of accented CD158a receptor expression in NK-lymphocytes in women with reproductive failures. Lik Sprava: 86-93.

52. Cohen BM, Machupalli S (2015): Use of Gammaglobulin to Lower Elevated Natural Killer Cells in Patients with Recurrent Miscarriage. J Reprod Med 60: 294-300.

53. Sacks G (2015): Enough! Stop the arguments and get on with the science of natural killer cell testing. Hum Reprod 30: 1526-1531 
54. Moffett A, Shreeve N (2015): First do no harm: uterine natural killer (NK) cells in assisted reproduction. Hum Reprod 30: 1519-1525.

55. Ramos-Medina R, García-Segovia Á, León JA, et al. (2013): New decision-tree model for defining the risk of reproductive failure. Am J Reprod Immunol 70: 59-68.

56. Katano K, Suzuki S, Ozaki Y, et al. (2013): Peripheral natural killer cell activity as a predictor of recurrent pregnancy loss: a large cohort study. Fertil Steril 100: 1629-1634.

57. Hiby SE, Walker JJ, O'shaughnessy KM, et al. (2004): Combinations of maternal KIR and fetal HLA-C genes influence the risk of preeclampsia andreproductive success J Exp Med 200: 957-965.

58. Yougbaré I, Tai WS, Zdravic D, et al. (2017): Activated NK cells cause placental dysfunction and miscarriages in fetal alloimmune thrombocytopenia. Nat Commun 8: 224. 